\title{
Time for helping
}

\section{Anastasia Danilov' ${ }^{1}$ Timo Vogelsang 2}

Received: 28 November 2014/ Accepted: 30 November 2015/Published online: 19 December 2015

(C) Economic Science Association 2015

\begin{abstract}
This study investigates whether individuals engage in prosocial behavior when it requires an investment of their time, but not money. In a laboratory experiment with rigorous anonymity arrangements, senders receive their payoff at the beginning. They may then engage in a tedious task to increase the earnings of exogenously disadvantaged recipients who otherwise receive no earnings. We find that senders are willing to sacrifice time to benefit recipients. Whether or not the recipient is present in the laboratory during the working time does not alter this decision. However, in a treatment variation some senders also display antisocial behavior.
\end{abstract}

Keywords Laboratory experiment - Social preferences · Time · Opportunity costs $\cdot$ Dictator game $\cdot$ Non-monetary giving

JEL Classification C91 $\cdot$ D64 $\cdot$ J22

Electronic supplementary material The online version of this article (doi:10.1007/s40881-015-0020-4) contains supplementary material, which is available to authorized users.

Timo Vogelsang

vogelsang@wiso.uni-koeln.de

Anastasia Danilov

danilov@wiso.uni-koeln.de

1 Department of Corporate Development and Business Ethics, University of Cologne, Albertus-Magnus-Platz, 50923 Cologne, Germany

2 Department of Personnel Economics and Human Resource Management, University of Cologne, Albertus-Magnus-Platz, 50923 Cologne, Germany 
Time is life itself....

Michael Ende (Momo, 1973).

\section{Introduction}

Numerous experimental studies on prosocial or other-regarding behavior focus on sharing monetary resources. One popular example is monetary giving in dictator games (Forsythe et al. 1994). In this game, a dictator receives a monetary endowment of $E$, whereas his counterpart (recipient) gets no initial endowment. The dictator can give away any amount of money $X \in[0, E]$ to the recipient. Studies typically find that fewer than $20 \%$ of dictators keep everything, and those who give transfer, on average, $20 \%$ of their endowment to recipients (Camerer and Colin 2003: 57). This observation suggests that subjects do not necessarily maximize their own monetary payoff but also care about the welfare of others. However, when the experimental procedures are double-blind $^{1}$ and, therefore, highly anonymous, the proportion of dictators who share their endowment abates, with more than $50 \%$ of dictators giving nothing. ${ }^{2}$

In naturally occurring contexts outside of the laboratory, other-regarding behavior is not restricted to money but can require sufficient time investments. Time, in general, is a limited personal resource that shapes our schedules and actions. It imposes constraints that, together with our preferences, largely determine what we do and how long we do it (Becker 1965). An important difference to money is that time is not fungible (Leclerc et al. 1995). It is not storable and cannot be saved or gained; however, like monetary resources it is a scarce resource. Even in situations where it is common to speak about "saved" or "gained" time, these words do not have the same meaning as when used in reference to money. The ubiquity of time and the differences to money make it important to study individuals' willingness to sacrifice time for the benefit of others.

In this study, we bring the time domain into the laboratory and focus on time as a medium of exchange in the context of other-regarding behavior. We used a simple experimental setting to investigate the act of giving by making subjects perform a task that is arduous and time-consuming. In our experiment, subjects placed in the role of senders received their payoff of $€ 10$ at the very beginning of the experiment. They could then perform a tedious task. The task resulted in no monetary costs or benefits for the sender but increased earnings of an anonymous and monetarily disadvantaged recipient who had no opportunity to increase her payoff. The treatments differed in the time recipients had to spend in the laboratory and their marginal monetary benefit from senders' effort (2-by-2 design). Additionally, we used a procedure that provides strict anonymity, thereby ruling out reputational concerns and peer pressure (Bénabou and Tirole 2006; Falk and Ichino 2006). Importantly, senders could end the experiment and leave the laboratory at any time without being seen by the experimenter.

\footnotetext{
1 In other words, "no one including the experimenter or any subsequent observer of the data could possibly know any subject's decision" or identity (Hoffman et al. 1996: 653).

2 A meta-study on dictator games can be found in Engel (2011).
} 
We find that senders are willing to spend time in the laboratory. In treatments where spending time results in a monetary benefit for the recipient, senders help the recipient to earn more regardless of whether or not the recipient is required to be concurrently present in the laboratory. When the senders' stay in the laboratory does not create any return for the recipient, the majority of subjects immediately leave the experiment. However, the opportunity to hold the recipient in the laboratory, even though this solely wastes the senders' and the recipients' time, provokes antisocial behavior from some senders. The results of the post-experimental questionnaire further suggest that senders' opportunity costs of time are correlated with the period spent in the laboratory.

A few other studies, for example, Carpenter and Myers (2010), Linardi and McConnell (2011), Cappellari et al. (2011), observe decision contexts with time at stake. They show the differences between time and monetary gifts and explain motivations for volunteering, which is a real-world example of time-giving. ${ }^{3}$ Ellingsen and Johannesson (2009) use time as an investment medium in an ultimatum game and show that a loss of time is more readily accepted than a loss of money. Comparing risk preferences for money and time, Bruyneel et al. (2015) find no difference. Furthermore, whereas subjects value losses of time and money differently, both mediums are treated equally in terms of the loss aversion parameters as well as the gains offered by both time and money.

Only two related studies experimentally investigate social preference situations with time as a medium of exchange. Berger et al. (2012) present an ultimatum game on waiting times, with the focus on different levels of anonymity. Noussair and Stoop (2015) focus on the difference between dictator, ultimatum, and trust games with time. In the dictator game, before receiving their earnings, dictators could decide how to distribute the necessary waiting time between themselves and receivers. The authors find that dictators are willing to take over between 30 and $40 \%$ of waiting time for the recipients.

While some of our findings are in line with these observations, our experimental protocol has substantial differences. First, it includes the addition of a tedious realeffort task demanding full attention. Second, one party can sacrifice his or her own time to increase the other party's income. Third, we vary the dimension of the impact of the working time and the presence of the recipient in the laboratory to shed more light on the underlying behavior. Fourth, in our experiment, subjects decide about time investment on a continuous basis, i.e., not once at the beginning of the experiment but continuously during their whole stay in the laboratory.

To our knowledge, no other experimental studies have looked at a context where time sacrifices - rather than monetary gifts—must be made in order to help increase a disadvantaged party's payoff. Hence, our study contributes to the substantially undervalued methodological literature on time as a decision medium and provides first insights into the role of opportunity costs for this decision.

\footnotetext{
3 In a recent and related study, Craig et al. (2014) focus on the effect of waiting periods before individuals can donate blood. We look, however, at the duration of the prosocial activity as such.
} 


\section{Experimental design and procedures}

The experiment was fully computerized using the software z-Tree (Fischbacher 2007) and conducted in the Cologne Laboratory of Economic Research. In total, 196 subjects participated in four treatments. All subjects were recruited at the University of Cologne using the online recruiting system ORSEE (Greiner 2015). The average age of the decision makers was 23.6 years, and $57.7 \%$ of them were females. Subjects had, on average, previously participated in 2.5 laboratory experiments. The invitation for the experiment stated that the experiment could last from $15 \mathrm{~min}$ up to $2 \mathrm{~h}$ and $15 \mathrm{~min}$. Furthermore, we invited groups of 4-8 participants in 15-min phases to make sure that new subjects constantly entered and exited the laboratory. ${ }^{4}$ The registration took place outside the laboratory. Subjects who arrived early could immediately proceed to their individual cubicles inside the laboratory. This phasedentry procedure (also explicitly mentioned in the experimental instructions) prevented subjects from learning how long other individuals stayed in the laboratory, thereby minimizing peer and reputational effects.

Upon entering the laboratory, the subjects took a seat in a randomly assigned, separated cubicle with the assigned role of either sender (labeled as Participant A) or recipient (labeled as Participant B). Senders found an envelope with $€ 10$ under their keyboard. The instructions on the computer screen explicitly told the senders to take (and keep) the money. Importantly, we used a series of control questions at the beginning of the experiment to completely ensure that subjects correctly understood the treatments.

Recipients received only a mandatory minimum show-up fee of $€ 2.50$ and had no further initial earnings in the experiment. The senders could stay in the laboratory and perform the task of entering the number " 1122334455 " in 15 -s intervals on the computer screen. ${ }^{5}$ For each correct entry, the respective recipient received a piece rate that varied between treatments. Additionally, after each correct entry, the screen was blocked for $15 \mathrm{~s}$. In the event of a wrongly typed-in number, an error message appeared stating that the number was wrong and needed to be corrected. ${ }^{6}$

Senders could end the experiment any time by clicking an "EXIT"-button on the screen. Afterward senders were asked some post-experimental questions about the experimental task, their demographic characteristics, and their opportunity costs of time. Senders left the laboratory after the survey was completed.

We elicited senders' opportunity costs of time with an incentivized question about the minimum wage they would be willing to accept in exchange to performing the experimental task for $1 \mathrm{~h}$. To incentivize this willingness to accept (WTA), we drew a random number and compared it to the stated wages. Then we randomly selected one sender from all senders in each treatment whose requested wage was

\footnotetext{
${ }^{4}$ In treatments where only a sender was present in the laboratory, we used groups of 4 to ensure a consistent number of senders in each group.

5 See online appendix for a screenshot of this stage.

6 We also used a test screen in the introduction to make senders familiar with this type of entering task and to rule out curiosity.
} 
below this number and invited him or her to work on the task in exchange for the amount he or she stated as the minimum wage on any convenient day. ${ }^{7}$

The exit of the laboratory was arranged through a different door that was not visible to an experimenter at the registration desk. No experimenter was present in the laboratory for the whole experiment. A second experimenter only entered the laboratory from time to time to privately instruct recipients whose senders had finished the experiment to proceed with the questionnaire and to deliver their payoffs. $^{8}$

The number-entering task required no special skills and was rather boring. The participants had to be attentive and were not able to do anything apart from focusing on the screen. We thus believe that all subjects should have been able to perform it and (not) enjoy it to the same extent. The only considerable difference among senders might be due to different opportunity costs of time.

Table 1 summarizes the treatments conducted.

In the ReLab5 treatment, each sender was matched with one anonymous recipient. The senders could contribute to the payoff of their recipients by staying in the laboratory and typing in the required number. For each correct entry, $€ 0.05$ was credited to the recipient's account. Considering 15-s waiting time before the entries, the senders could increase recipients' earnings by up to $€ 12$ per hour (compared with an average student job wage of around $€ 10$ per hour). The recipients had to stay in the laboratory for 15 min longer than their corresponding senders and received a show-up fee of $€ 2.50$ for this. Consequently, senders and recipients left the laboratory separately. ${ }^{9}$

In the NoReLab5 treatment, everything stayed the same as in the ReLab5 treatment except that recipients were not in the laboratory during the time the senders were working. The senders were informed that the recipient would attend the experiment on a different day only for $15 \mathrm{~min}$ and receive an initial show-up fee of $€ 2.50$ plus whatever earnings the sender generates for her. Therefore, the recipients spend no additional time for the payoff generated by the senders. This treatment allows us to control for whether the senders take the recipients' opportunity costs into account. If they think that $15 \mathrm{~s}$ of the recipient's waiting time are worth more than $€ 0.05$, then the duration of stays in the laboratory should be, ceteris paribus, higher in the NoReLab5 than in the ReLab5 treatment. Additionally, time spent by senders can be considered as more prosocial and/or efficient than in ReLab5. Conversely, it is feasible to argue that working time could be reduced because the receiver does not "share" time costs with the sender, and, therefore, less help might be especially easy to justify.

\footnotetext{
7 We chose to pay the stated minimum wage and not the random number for simplicity reasons. An example in the instructions further explained the procedure.

${ }^{8}$ The instructions clearly stated that it would not be possible for the experimenter to connect the name of the subject with the decision and that no one will ever learn their identity.

${ }^{9}$ Recipients were permitted to use the Internet for private purposes while senders were working. Senders were informed about this detail in order to mitigate the possible argument that recipients had a difficult time in the laboratory and it was not worth the earnings. Wilson et al. (2014) support this argument by showing that doing anything is preferable to doing nothing in experiments.
} 
Table 1 Overview of treatments

\begin{tabular}{lllll}
\hline Treatment & $\begin{array}{l}\text { Sender increases the } \\
\text { recipient's payoff by... }\end{array}$ & $\begin{array}{l}\text { Recipient stays in the } \\
\text { laboratory while sender is } \\
\text { working }\end{array}$ & $\begin{array}{l}\text { N of } \\
\text { subjects }\end{array}$ & $\begin{array}{l}\text { N of independent } \\
\text { observations } \\
\text { (senders) }\end{array}$ \\
\hline ReLab5 & $€ 0.05$ per click & Yes & 64 & 32 \\
NoReLab5 & $€ 0.05$ per click & No & 64 & 32 \\
ReLab0 & $€ 0$ per click & Yes & 42 & 21 \\
NoReLab0 & $€ 0$ per click & No & 26 & 26 \\
\hline
\end{tabular}

Two "No Impact Treatments" serve as a control for alternative explanations for why senders might stay in the laboratory other than to provide monetary help for the recipients, for example cases in which subjects hesitate to leave the laboratory early, feel an obligation to work on the task, choose to spend their spare time in the experiment, or would like to exercise control over the recipient and his or her time in the laboratory.

In the ReLab0 treatment, the setup was the same as in the ReLab5 treatment, except that the working time of the sender had no monetary impact on the payoff of the recipient. In other words, the piece rate for every entered number was 0 . Nevertheless, the recipients had to stay in the laboratory as long as the senders were working. Thus, prosocial senders should have left the laboratory as soon as possible because not only was their action of no monetary impact, but it also kept the recipient in the laboratory.

In the NoReLab0 treatment, the piece rate was 0 , but there was no recipient in the laboratory. Similar to the ReLab0, senders could choose to work on a task with no purpose.

Together these four treatments comprised a 2-by-2 design, where the presence of the recipient in the laboratory varied on one dimension and the impact factor of the sender's time on the other.

\section{Manipulation check}

In the post-experimental questionnaire, subjects evaluated different statements about the experimental task on a 7-point Likert scale with 1 equal to "strongly disagree" and 7 "strongly agree." 10 The data show that the experimental design worked as desired. Subjects report low enjoyment in performing the task and low shame in leaving the experiment as compared to the neutral response of 4 (mean $=2.7, \mathrm{SDs}=1.9, N=111, p$-values $<0.001, z=-5.985$ and $z=6.174$, respectively, two-sided Wilcoxon signed-rank test). Subjects also did not "work on the task because they had nothing else to do" (mean $=3.4, \mathrm{SD}=1.9, N=111, p$ -

${ }^{10}$ See online appendix for the precise statements. 
value $=0.003, z=-3.012$, two-sided WSR test). There are virtually no betweentreatment differences in these measures. ${ }^{11}$

\section{Data and specification}

In the following, the working time is determined as the number of correct entry times $15 \mathrm{~s}$, which is then converted into minutes. Figure 1 depicts the cumulative distribution functions of senders' time spent in the laboratory. The distribution of working times is very similar for the ReLab5 and NoReLab5 treatments, in which working had a monetary impact for the recipient, and differs visibly from the control conditions ReLab0 and NoReLab0. However, almost $15 \%$ of subjects spent more time in the ReLab0 treatment than in any other treatment. Regression analysis provides statistical support for these findings. We present different models (Tables 2, 3) with the working time (in minutes) as the dependent variable. ${ }^{12}$ However, as senders could decide at any stage during their working time whether they want to continue to work or leave, the semi-parametric Cox proportional hazard model, similar to Rosaz et al. (2014), is the most appropriate econometric approach given the continuous decision to quit that senders had. ${ }^{13}$ The other models serve as a robustness check and provide additional analyses of the data to address different questions.

\section{Results}

\subsection{Result 1a: senders spend time to increase recipients' payoffs}

In the ReLab5 treatment, the senders stayed in the laboratory for an average of $23.56 \mathrm{~min}(\mathrm{SD}=15.34)$, thereby generating an average of $€ 4.71$ for the recipients. The data show that with $23.34 \mathrm{~min}(\mathrm{SD}=15.54)$ the senders worked similarly long in the NoReLab5 treatment. This is supported by the low effect size of 0.014 and can also be seen in the similar distributions of working times in Fig. 1. There is no significant difference between these two treatments ( $p$-value $=0.946$, two-sided Mann-Whitney $U$ test). A power analysis with a significance level of 0.05 and a power of 0.80 reveal that such an economically insignificant treatment difference would be statistically significant only in a very large sample of 76,377 or more

\footnotetext{
11 The only difference we find between treatments is regarding the enjoyment of the task. The enjoyment of the task is significantly higher in the ReLab5 and NoReLab5 treatments (mean $=3.1, \mathrm{SD}=2$, $N=64$ ) than in the "No Impact Treatments" ReLab0 and NoReLab0 treatments (mean $=2.2$, $\mathrm{SD}=1.5, N=47, p$-value $=0.011$, two-sided MWU test). This can be due to the positive effect of senders' activity on recipients' earnings. Separate tests for each treatment report similar results as reported in the text for the pooled sample. All $p$-values are below 0.0836 , two-sided WSR test with one exception for the statement whether subjects worked because they had nothing else to do in the NoReLab0 treatment. Here, the answers are not different from the neutral response (mean $=4, \mathrm{SD}=2.1$ and a two-sided WSR $p$-value is 0.9795). See Table 6 in the online appendix for more details.

12 Please see the online appendix for both tables without control variables. No notable difference occurs.

13 We thank the editor for this suggestion.
} 


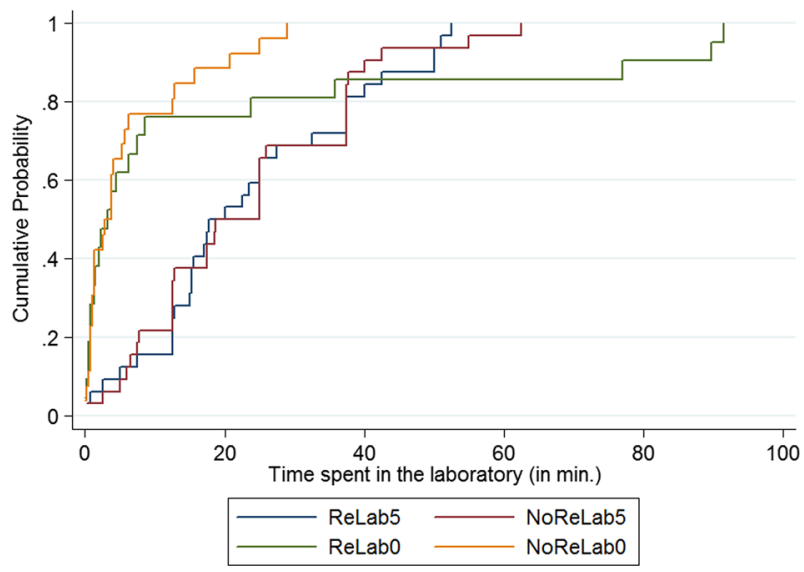

Fig. 1 Cumulative distribution functions of time spent by the senders

Table 2 Working time

\begin{tabular}{|c|c|c|c|c|c|c|}
\hline & \multicolumn{2}{|c|}{$\begin{array}{l}\text { Cox PH model } \\
\text { (decision to leave the } \\
\text { laboratory at a specific } \\
\text { point in time) }\end{array}$} & \multicolumn{2}{|c|}{$\begin{array}{l}\text { OLS (working time in } \\
\text { minutes) }\end{array}$} & \multicolumn{2}{|c|}{$\begin{array}{l}\text { Probit (probability } \\
\text { of working time } \\
\geq 10 \mathrm{~min} \text { ) }\end{array}$} \\
\hline & (1) & (2) & (3) & (4) & (5) & (6) \\
\hline No recipient in the laboratory & $\begin{array}{l}1.33 \\
(0.27)\end{array}$ & $\begin{array}{l}2.64 * * * \\
(0.89)\end{array}$ & $\begin{array}{l}-4.37 \\
(3.48)\end{array}$ & $\begin{array}{l}-11.59 * * \\
(5.31)\end{array}$ & $\begin{array}{l}-0.05 \\
(0.11)\end{array}$ & $\begin{array}{l}-0.05 \\
(0.17)\end{array}$ \\
\hline Monetary impact & $\begin{array}{l}0.43 * * * \\
(0.09)\end{array}$ & $\begin{array}{l}0.79 \\
(0.25)\end{array}$ & $\begin{array}{l}12.21 * * * \\
(3.50)\end{array}$ & $\begin{array}{l}5.44 \\
(5.13)\end{array}$ & $\begin{array}{l}0.68 * * * \\
(0.11)\end{array}$ & $\begin{array}{l}0.68 * * * \\
(0.17)\end{array}$ \\
\hline $\begin{array}{l}\text { No recipient in the laboratory* } \\
\text { monetary impact }\end{array}$ & & $\begin{array}{l}0.31 * * \\
(0.14)\end{array}$ & & $\begin{array}{l}12.84^{*} \\
(7.18)\end{array}$ & & $\begin{array}{l}-0.01 \\
(0.23)\end{array}$ \\
\hline Controls & Yes & Yes & Yes & Yes & Yes & Yes \\
\hline$N$ & 111 & 111 & 111 & 111 & 111 & 111 \\
\hline Log likelihood & -409.661 & -406.202 & & & & \\
\hline $\operatorname{Adj} R^{2}$ & & & 0.098 & 0.117 & & \\
\hline Pseudo $R^{2}$ & & & & & 0.309 & 0.309 \\
\hline
\end{tabular}

The table reports results from different regressions. Columns 1 and 2 estimate the semi-parametric Cox proportional hazard model, columns 3 and 4 report results from an OLS regression, and columns 5 and 6 use a probit model. Standard errors are reported in parentheses. The hazard ratios are presented as coefficients in the survival models. Marginal effects are shown for the probit model. "No recipient in the laboratory" is a binary variable equal to 1 in the treatments where recipients were not present in the laboratory (in NoReLab5 and NoReLab0) and 0 otherwise (in ReLab5 and ReLab0). "Monetary impact" is a binary variable equal to 1 in the treatments (ReLab5 and NoReLab5) and 0 otherwise. Control variables include age, gender, and whether or not subjects are studying economics

$* p<0.1$; ** $p<0.05$; *** $p<0.01$ 
subjects per treatment. Although the recipient in the NoReLab5 treatment did not share the amount of time in the laboratory, there is no difference in time spent by the senders.

What alters the decision, however, is the impact of their time on the recipient's monetary payoff. Column 1 of Table 2 presents the main effects. The hazard of leaving the laboratory at a specific point in time, holding the presence of the recipient constant, is $57 \%$ lower if working has an impact compared to if working has no impact ( $p$-value $<0.001$ ). Column 2 presents the estimation for the full model. The interaction term shows that if there is no recipient in the laboratory, there is a highly significant ( $p$-value $=0.01$ ) difference between the cases in which working has an impact or not. The OLS regressions (columns 3 and 4) provide qualitatively identical results, which serve as a robustness check. These results indicate that the monetary impact had a significant effect when the recipient was not in the room but had no significant effect when the recipient was present.

\subsection{Result 1b: ...however, senders also spend time even if they cannot increase recipients' payoffs}

In the control "No Impact Treatments" (NoReLab0 and ReLab0), the majority, $77 \%$ of individuals, left the laboratory within the first $10 \mathrm{~min}^{14}$

Yet a non-negligible amount of individuals stayed longer although working had no monetary impact. Here the presence of the recipient matters. The absence of the recipient in the "No Impact Treatments" (NoReLab0) causes the senders to quit more quickly. A sender under these circumstances who is still in the laboratory by a certain time has an almost tripled (164\% higher) hazard of leaving the laboratory at the next moment compared to the "No Impact Treatment" with a recipient (ReLab0) (Table 2, column 2, p-value=0.004). Having the possibility to act antisocial, which is only possible in the ReLab0 treatment, induces some subjects into such a behavior. This is very unlikely to be random, and furthermore, it is not an insignificant fraction with three subjects working longer than in any other treatment $(>70 \mathrm{~min}){ }^{15}$

\subsection{Result 2: senders are more likely to stay in the laboratory for more than $10 \mathrm{~min}$ if they can increase the recipients' payoff}

The above results suggest that senders spend some time regardless of whether their time positively impacts the recipient. The probability of working on the task more than $10 \mathrm{~min}$, however, increases significantly by $68 \%$ when working has a monetary impact compared to no monetary impact (Table 2, column 5 , $p$-value $<0.001)$. Furthermore, this does not significantly differ whether or not the recipient is in the laboratory (Table 2 , column $6, p$-value $=0.977$ ). There might be a variety

\footnotetext{
${ }^{14}$ We define a 10-min threshold because it seems reasonable that everything below 10 min could be attributed to a variety of reasons for not leaving the laboratory (curiosity, confusion, shyness, demand).

15 The probability that these three subjects together show up by chance in the ReLa0 condition (if they are equally likely to show up in any of the treatments $)$ is $(21 / 111) \times(20 / 110) \times(19 / 109)=0.006$.
} 
of reasons why senders stay in the laboratory a couple of minutes, but whether or not staying has a prosocial impact is a significant factor for staying longer.

\subsection{Result 3: the higher the sender's opportunity cost, the less she works}

Making use of the elicited opportunity costs of time (WTA), we look at whether the senders' opportunity costs (Mean $=17.43, \mathrm{SD}=11.52$ ) are correlated with the working time. ${ }^{16}$

Table 3 shows that the WTA is significantly correlated in all of the specifications (all $p$-values $<0.013$ ). Model (2) shows, for instance, that a $1 €$ increase in senders' WTA is associated with a $3 \%$ higher hazard of leaving the laboratory.

Interestingly, all 3 subjects who stayed more than $70 \mathrm{~min}$ in the ReLab0 treatment had a WTA of less than 12 (which would be a possible hourly wage for the recipient in the experiment). Antisocial behavior may thus be only present if it is less costly. ${ }^{17}$

Table 3 Opportunity costs and working time

\begin{tabular}{|c|c|c|c|c|}
\hline & \multicolumn{2}{|c|}{$\begin{array}{l}\text { Cox PH model (decision to } \\
\text { leave the laboratory at } \\
\text { each time) }\end{array}$} & \multicolumn{2}{|c|}{$\begin{array}{l}\text { OLS (working time in } \\
\text { minutes) }\end{array}$} \\
\hline & (1) & (2) & (3) & (4) \\
\hline Sender's WTA & $\begin{array}{l}1.03 * * * \\
(0.01)\end{array}$ & $\begin{array}{l}1.03 * * * \\
(0.01)\end{array}$ & $\begin{array}{l}-0.50 * * * \\
(0.15)\end{array}$ & $\begin{array}{l}-0.50 * * * \\
(0.15)\end{array}$ \\
\hline No recipient in the laboratory & $\begin{array}{l}1.13 \\
(0.24)\end{array}$ & $\begin{array}{l}2.10 * * \\
(0.73)\end{array}$ & $\begin{array}{l}-3.43 \\
(3.33)\end{array}$ & $\begin{array}{l}-10.76^{* *} \\
(5.06)\end{array}$ \\
\hline Monetary impact & $\begin{array}{l}0.45^{* * * *} \\
(0.09)\end{array}$ & $\begin{array}{l}0.74 \\
(0.23)\end{array}$ & $\begin{array}{l}10.04 * * * \\
(3.40)\end{array}$ & $\begin{array}{l}3.15 \\
(4.93)\end{array}$ \\
\hline No recipient in the laboratory* monetary impact & & $\begin{array}{l}0.36 * * \\
(0.16)\end{array}$ & & $\begin{array}{l}13.04 * \\
(6.83)\end{array}$ \\
\hline Controls & Yes & Yes & Yes & Yes \\
\hline$N$ & 111 & 111 & 111 & 111 \\
\hline Log likelihood & -402.291 & -399.720 & & \\
\hline $\operatorname{Adj} R^{2}$ & & & 0.179 & 0.200 \\
\hline
\end{tabular}

The table reports results from a survival analysis using the semi-parametric Cox proportional hazard model and also from OLS regressions. Standard errors are reported in parentheses. The hazard ratios are presented as coefficients in the survival models. "Recipient in the laboratory" is a binary variable equal to 1 in the treatments where recipients were present in the laboratory (in ReLab5 and ReLab0) and 0 otherwise (in NoReLab5 and NoReLab0). "No monetary impact" is a binary variable equal to 1 in the treatments (ReLab0 and NoReLab0) and 0 otherwise. WTA refers to the minimum amount the sender was willing to accept for working for $1 \mathrm{~h}$ on the task. Control variables include age, gender, and whether or not subjects are studying economics

$* p<0.1 ; * * p<0.05 ; * * * p<0.01$

\footnotetext{
${ }^{16}$ We also elicited the belief about the time costs of the recipient. However, this happened ex-post and were not incentivized. Statistics are shown in the online appendix.

17 See the online appendix for more detailed statistics.
} 


\section{Conclusion}

This study addresses the question of whether individuals will spend time in order to help to increase the earnings of an exogenously disadvantaged recipient. Moreover, it considers the role played by opportunity costs of time.

The sender's payoff remains unchanged and is paid before the beginning of the experiment. The task is tedious and time-consuming. The strict anonymity arrangements, freedom to leave the laboratory at any time, and a task which is equally demanding for all subjects are important features of our experiment. Furthermore, this design and procedure rule out concerns about reputation and peer effects, leaving no room for signaling one's generosity.

We find that senders generate money for recipients and therefore spend a nonnegligible amount of time in the laboratory. This holds regardless of the time invested by the receiving party. Unlike the findings of Hoffman et al. (1996), rigorous anonymity conditions do not provoke egoistic behavior in the treatments in which spending time has a monetary impact, supporting the findings of Berger et al. (2012).

The data further show that the presence of the recipient in the laboratory is important if working has no monetary impact. Some senders are willing to spend their time working in the laboratory even when recipients do not benefit but are required to stay in the laboratory for the same amount of time. This evidence for antisocial behavior is notable because senders not only destroy others time but also their own. Senders' working time is further correlated with their incentivized opportunity costs.

While sharing and destroying monetary resources in the laboratory is already a part of the conventional wisdom, we provide clean evidence that prosocial and antisocial behavior is also present when it requires time expenditures.

Acknowledgments We thank Rainer M. Rilke, Robert Slonim, Jan Stoop, Sebastian Tonke, and two anonymous referees for helpful comments. We also thank Prof. Dr. Dirk Sliwka, Department of Personnel Economics and HRM, and Prof. Bernd Irlenbusch, Department of Corporate Development and Business Ethics, University of Cologne for financial support.

\section{References}

Becker, G. S. (1965). A theory on the allocation of time. The Economic Journal, 75(299), 493-517.

Bénabou, R., \& Tirole, J. (2006). Incentives and prosocial behavior. American Economic Review, 96(5), 1652-1678.

Berger, R., Rauhut, H., Prade, S., \& Helbing, D. (2012). Bargaining over waiting time in ultimatum game experiments. Social Science Research, 41(2), 372-379.

Bruyneel, S., Dewitte, S., Diecidue, E., Festjens, A. (2015) Time-based versus money-based decision making under risk: an experimental investigation. Journal of Economic Psychology 50:52-72

Camerer, C. F. (2003). Behavioral game theory: Experiments in strategic interaction (Vol. 32). Princeton: Princeton University Press. 
Cappellari, L., Ghinetti, P., \& Turati, G. (2011). On time and money donations. The Journal of SocioEconomics, 40(6), 853-867.

Carpenter, J., \& Myers, C. K. (2010). Why volunteer? Evidence on the role of altruism, image, and incentives. Journal of Public Economics, 94(11-12), 911-920.

Craig, A., Carbarino, E., Heger, S. A., \& Slonim, R. (2014). Waiting to give. IZA discussion paper no. 8491 .

Ellingsen, T., \& Johannesson, M. (2009). Time is not money. Journal of Economic Behavior and Organization, 72(1), 96-102.

Engel, C. (2011). Dictator games: A meta study. Experimental Economics, 14(4), 583-610.

Falk, A., \& Ichino, A. (2006). Clean evidence on peer effects. Journal of Labor Economics, 24(1), 39-58.

Fischbacher, U. R. S. (2007). z-Tree: Zurich toolbox for ready-made economic experiments. Experimental Economics, 10(2), 171-178.

Forsythe, R., Horowitz, J., Savin, N. E., \& Sefton, M. (1994). Fairness in simple bargaining experiments. Games and Economic Behavior, 6(3), 347-369.

Greiner, B. (2015). Subject pool recruitment procedures: Organizing experiments with ORSEE. Journal of the Economic Science Association, 1(1), 114-125.

Hoffman, E., McCabe, K., \& Smith, V. L. (1996). Social distance and other-regarding behavior in dictator games. American Economic Review, 86(3), 653-660.

Leclerc, F., Schmitt, B. H., \& Dubé, L. (1995). Waiting time and decision making: Is time like money? Journal of Consumer Research, 22, 110-119.

Linardi, S., \& McConnell, M. A. (2011). No excuses for good behavior: Volunteering and the social environment. Journal of Public Economics, 95(5-6), 445-454.

Noussair, C., \& Stoop, J. (2015). Time as a medium of reward in three social preference experiments. Experimental Economics, 18(3), 442-456.

Rosaz, J., Slonim, R., \&Villeval, M. C. (2014). Peer effects at work. IZA discussion paper no.6475.

Wilson, T. D., Reinhard, D. A., Westgate, E. C., Gilbert, D. T., Ellerbeck, N., Hahn, C., et al. (2014). Just think: The challenges of the disengaged mind. Science, 345(6192), 75-77. 